\title{
SOFTWARE AND HARDWARE COMPLEX FOR SETTING OF AUTOMATIC EXCITATION REGULATORS OF TURBOGENERATORS
}

\author{
Venera A. Sulaymanova ${ }^{1, *}$, Yuri S. Borovikov ${ }^{1}$, Mikhail V. Andreev ${ }^{1}$, and \\ Aleksey A. Suvorov ${ }^{1}$ \\ ${ }^{1}$ Tomsk Polytechnic University, 634050, Tomsk, Russia
}

\begin{abstract}
The motivation of the presented research is based on the needs for development of new methods and tools for research setting problem of automatic excitation regulators of turbogenerators. Simulation tools must meet the requirements of reproduction processes reliability in all elements of electric power system. The developed block diagram of the adequate mathematical model of automatic excitation regulator is presented. The simulation results of electric power system scheme confirm the adequacy of the reproduction processes of functioning of automatic excitation regulator and a generator.
\end{abstract}

\section{Introduction}

In view of the interconnection of all elements of electric power system (EPS) in a single continuous process of generation, transmission, distribution and consumption of electric power, any changes of mode parameters (load change, connection and disconnection of equipment, short circuits and others) influence on functioning of the power equipment and EPS as a whole. The basic elements of maintaining required voltage level, increasing the stability of EPS generators operating in parallel and damping of oscillation are automatic excitation regulators (AER) of generators. At the present time used AER with power system stabilizer (PSS) have a large number of tuning factors, and their choice has impact on reliable and stable EPS functioning. In this way, setting of AER is an important practical problem, which for many years many research groups engaged and engage.

Setting must be implemented for each generator considering of scheme-regime operational conditions in a scheme with its characteristics and parameters, and also EPS reaction on setting. Great importance in the study of this problem has reliability of used mathematical models of all EPS elements. Technical requirements for the excitation system (ES) and AER PSS of synchronous generators, and also a method for certification and setting of AER PSS are defined by the standard of Joint-stock Company "System Operator of the United Power System" [1].

\footnotetext{
${ }^{*}$ Corresponding author: venera20@,tpu.ru
} 


\section{Mathematical model of excitation system with an automatic excitation regulator with power system stabilizer}

Using developed in Tomsk Polytechnic University Hybrid Real-Time Simulator (HRTSim) $[2,3]$ of EPS is one of the most preferred tools for solving the problem of adequate setting of AER. HRTSim of EPS is the soft- and hard-ware complex, comprising set of universal specialized processors for all kinds of power equipment of simulated EPS and control system.

In HRTSim of EPS the mathematical model of a synchronous machine represents full Park's-Gorev's system equations increased accuracy, in cooperation with the equations of forming three-phase system A, B, C and mutual conversion systems variables $\mathrm{d}$, q and A, $B$, C. Required reliability of simulation is provided by increasing number of simulated damping circuits (three d-axis and four q-axis) and accounting the frequency dependence of their parameters [4].

For adequate reproduction processes in EPS also it's necessary to have detailed mathematical models of the auxiliary EPS equipment: ES, primary engines, driven mechanisms and others. Developed structure of hybrid processor HRTSim of EPS [5] allows to realize any kinds of AER. The block diagram of the mathematical model of ES with AER PSS is presented in figure 1.

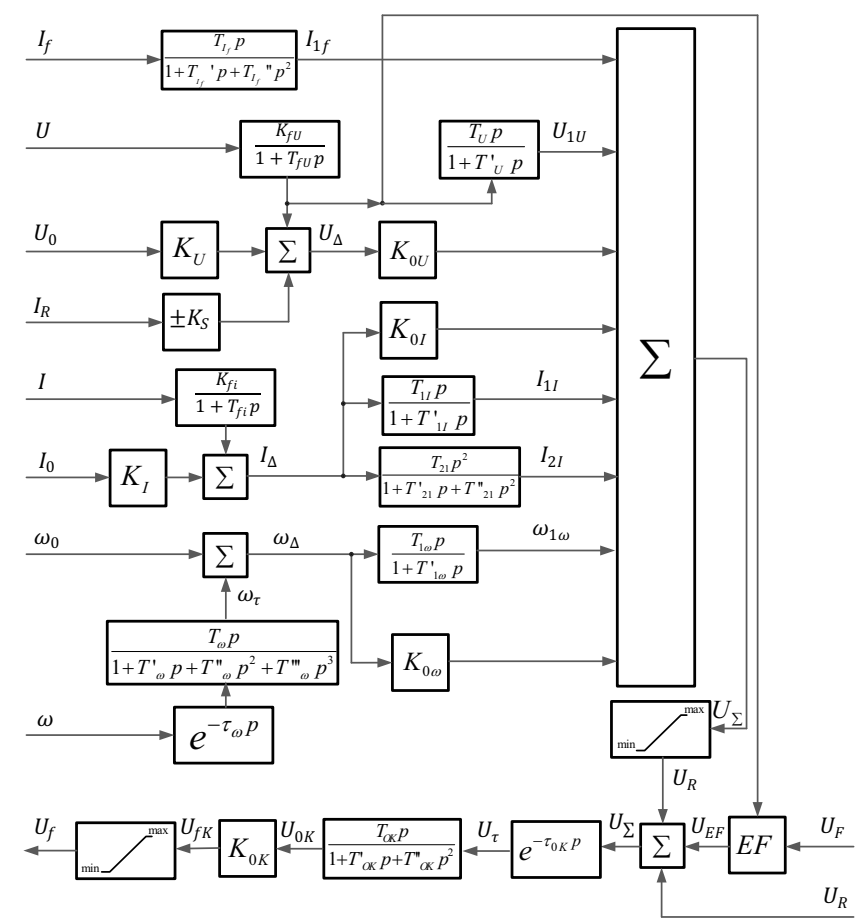

Fig. 1. Block diagram of the mathematical model of ES with different kinds of AER PSS.

Presented block diagram takes into account significant factors essential for adequate reproduction, namely the specifics of such ES and AER and inherent control channels, tuning factors, parameters and defining differential equations transfer functions: $K_{S}-\mathrm{a}$ static factor of voltage corrector of the stator $U, K_{U}$ - threshold factor of stator voltage $U, K_{I}$ - a compounding factor for the complete stator current $I$, EF - different types of 
excitation forcing, $K_{0 U}-$ a gain factor of control channel on the stator voltage deviation $U_{\Delta}, K_{0 \omega}-$ a factor of the control channel on the frequency deviation $\omega_{\Delta}$, blocked when the limit value, $W(p)=\frac{K_{f U}}{1+T_{f U} p}$ and $W(p)=\frac{K_{f i}}{1+T_{f i} p}-$ transfer functions of the rectifiers and voltage filters $U$ and stator current $I, W(p)=\frac{T_{U}}{1+T_{U}^{\prime} p}-$ the transfer function of the control channel on the derivative of the stator voltage $U_{1 U}, W(p)=\frac{T_{I_{f}}}{1+T_{I_{f}}^{\prime} p+T_{I_{f}}^{\prime \prime} p^{2}}-\mathrm{a}$ transfer function of the control channel on the derivative of the excitation current $I_{1 f}$, $W(p)=\frac{T_{1 \omega}}{1+T_{1 \omega}^{\prime} p}-\mathrm{a}$ transfer function of the control channel on the derivative of the frequency, $W(p)=e^{-\tau_{\omega} p} \frac{T_{\omega} p}{1+T_{\omega}^{\prime} p+T_{\omega}^{\prime \prime} p^{2}+T_{\omega}^{\prime \prime \prime} p^{3}}-$ a transfer function of the measurement unit and frequency conversion to form its deviation $\omega_{\Delta}, W(p)=e^{-\tau_{O K} p} \frac{T_{O K} p}{1+T_{O K}^{\prime} p+T_{O K}^{\prime \prime} p^{2}}-\mathrm{a}$ transfer function of total excitation control channel, including the rectifier, exciter and others, $K_{0 I}-$ a gain factor of control channel on the stator current deviation $I_{\Delta}$ from set value $I_{0}, W(p)=\frac{T_{1 I} p}{1+T_{1 I}^{\prime} p}-$ a transfer function of the control channel on the derivative of the stator current $I_{1}, W(p)=\frac{T_{2 I} p^{2}}{1+T_{2 I}^{\prime} p+T_{2 I}^{\prime \prime} p^{2}}-$ a transfer function of the control channel on the second derivative of the stator current $I_{2 I}$.

In figure 2 the fragment of simulated scheme of Tomsk EPS in HRTSim is given.

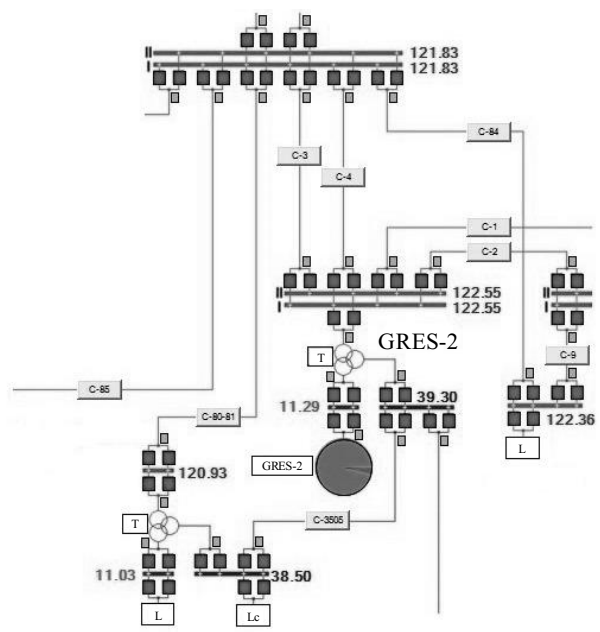

Fig. 2. Fragment of scheme of Tomsk EPS: GRES-2 - thermal power plant, L - load, Lc - controlled load, $\mathrm{T}$ - transformer, $\mathrm{C}$ - power transmission line. 


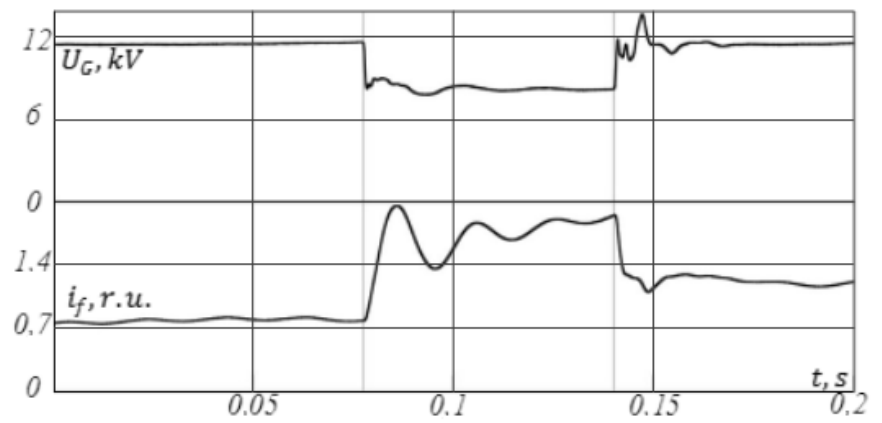

Fig. 3. Oscillograms of automatic voltage regulation process at the generator terminal GRES-2 for three-phase short circuit at the end of the line C-84.

The results of the experiment perform oscillograms of automatic voltage regulation by AER GRES-2 for three-phase short circuit at the end of the line C-84 (figure 3). Implemented in HRTSim of EPS oscillograms confirm the adequacy of the reproduction processes of functioning of AER PSS and generator, that allow to use this complex for solve setting of AER problem, as well as for other tasks, such as research regimes of EPS with the presence of compensating devices [6].

\section{Conclusion}

Efficiency of solution of AER setting problem depends on used mathematical models of AER, generators and all the other elements of the simulated EPS. Developed HRTSim allows to use detailed models of AER and other equipment for the problems of research and design of EPS. Developed and tested adaptable mathematical model of ES, allowing to simulate different types of AER PSS and ensuring reliable reproduction of the processes occurring in this equipment.

The work was supported by the Ministry of Education and Science of the Russian Federation under the governmental grant "Science" № 3901 (Research and development of the hybrid model of the back-to-back high-voltage direct current transmission system).

\section{References}

1. Standard of organization "Requirements for excitation systems and automatic excitation regulators with power system stabilizer of synchronous generators"

2. A. Suvorov, Y. Borovikov, A. Gusev, A. Sulaymanov, M. Andreev, N. Ruban, R. Ufa, Electrical Engineering (to be published)

3. N. Y. Ruban, A. S. Gusev, A. Y. Pishchulin, MATEC Web of Conf., 37, 01046 (2015)

4. V. M. Yakutov, Electricity, 2, 23 (1992) [in Russian]

5. Y. S. Borovikov, A. S. Gusev, A. O. Sulaymanov, R. A. Ufa, ISCAI, 148 (2014)

6. L. L. Balyberdin, E. Yu. Zmaznov, Yu. S. Kraichik, B. P. Krasnova, N. G. Lozinova, M. I. Mazurov, G. G. Prochan, Power Technology and Engineering, 38, 119 (2004) 